\title{
The Association of Irisin with the Progression of Diabetes Mellitus Irisin and Diabetes Progression ${ }^{\mathrm{i}}$
}

\author{
Nihal Yücel ${ }^{1, *}$, Aycan Bölük ${ }^{1}$, Özlem Madenci ${ }^{1}$, Zeynep Yildiz ${ }^{1}$, Mehmet Sargın², Asuman Orçun ${ }^{1}$ \\ ${ }^{1}$ Clinical Biochemistry Department, Lütfi Kurdar Kartal Training and Research Hospital, İstanbul, Turkey \\ ${ }^{2}$ Department, İstanbul, Lütfi Kırdar Kartal Training and Research Hospital, Diabetes Turkey
}

Copyright $\odot 2018$ by authors, all rights reserved. Authors agree that this article remains permanently open access under the terms of the Creative Commons Attribution License 4.0 International License

\begin{abstract}
Introduction: There is an ongoing debate over the involvement of irisin in the glucose metabolism. In this study, we aimed to see the association of irisin with the markers of glucose intolerance. Materials and Methods: We analyzed the relation of irisin with metabolic markers such as Homeostatic Model Assessment-Insulin Resistance (HOMA-IR), insulin, and glucose in 152 subjects, subdivided into normal, impaired glucose tolerance and diabetic subgroups. Results: We found that the irisin levels of individuals with different stages of glucose intolerance did not show any significant difference. There was not any correlation between irisin levels with HOMA-IR. However, we observed a positive and significant correlation between circulating irisin concentrations and 120-minute glucose, 120-minute insulin, and HbAlc levels. Conclusion: The conclusive results of this study are that irisin could be somehow associated with insulin resistance but do not show a significant difference with neither diabetes nor different stages of insulin resistance.
\end{abstract}

Keywords Irisin, Insulin Resistance, Diabetes

\section{Introduction}

There is increasing evidence about the secretory function of skeletal muscle (1). It releases numerous cytokines or other peptides that exert local or distant specific effects. (2) These cytokines called 'myokines' modulate metabolic processes such as metabolic syndrome, obesity, insulin resistance and type 2 diabetes (T2D), through their endocrine effects on other organs $(2,3)$. Therefore, skeletal muscle is now identified as an endocrine organ.

Irisin is a novel myokine derived from fibronectin type III domain containing 5 (FNDC5, also known FRCP2 and PeP). FNDC5 is a type I transmembrane protein of myocytes. This protein is proteolytically cleaved and secreted as irisin. (4). It is reported that FNDC5 and consequently irisin secretion is stimulated by peroxisome proliferator-activated receptor- $\gamma$ coactivator-1alfa (PGC1 alfa) (4). It is previously demonstrated that transgenic mice with mildly elevated muscle PGC1 alfa, are resistant to age-related obesity and diabetes and have a prolonged lifespan (5). In fact, PGC1 alfa acts through the irisin like substances released from skeletal muscle. Once secreted, irisin acts on the subcutaneous adipose tissue; induces browning and the expression of mitochondrial uncoupling protein-1 (UCP1), which in turn increases energy expenditure and thermogenesis. It was previously shown that brown adipose tissue modulates peripheral tissue metabolism by thermogenesis and enhance insulin sensitivity; consequently, increased brown adipose tissue (BAT) improves glucose tolerance (6). In their study, Boström and colleagues also showed that exercise caused a mild increase in blood irisin levels which increase the total body energy expenditure and obesity-linked insulin resistance (4). By these findings, it is postulated that irisin is related to exercise and involved in a variety of metabolic processes such as obesity, insulin resistance, and diabetes.

In recent years, several clinical studies, with contradicting results, have investigated the association of irisin with different metabolic situations. One of the most investigated conditions is exercise and irisin relation. However, data obtained from the molecular (mRNA induction of FNDC5/irisin) or clinical (circulation levels of irisin) human studies were controversial. Circulating irisin levels were found increased in some studies $(7,4)$, transiently increased in some others (8). There were also studies that found an acute increase after exercise but no change after long-term training (9). In their report on FNDC5 mRNA induction after different type of exercise, Pekkala found an increase in only one type of exercise and concluded that neither longer-term nor single exercise markedly increases skeletal muscle FNDC5 expression or serum irisin (10). Other studies did not confirm any difference before or after exercise $(11,12)$. Another study with gene expression arrays found an exercise-induced increase of muscle FNDC5 mRNA in older subjects but not 
in younger ones (13).

The correlation between irisin and obesity is also largely investigated. In fact, recent studies showed that, besides the muscle tissue, irisin is also secreted by white adipose tissue $(14,15)$. A large number of studies on the relation of irisin and body mass index (BMI) revealed conflicting results; some of them found a positive correlation between irisin and BMI (7, 16-18), while others showed no (19) or even negative correlation $(20,21)$.

The role of irisin in diabetes or insulin resistance is also one of the popular topics of the last few years. However, several studies carried on this issue have ended up with discrepant results. Some of them found a positive effect of irisin on glucose homeostasis $(16,20,22$,), some negative $(13,17,23,24)$, and some others no effect (25). There are quite a few studies reporting lower insulin concentrations in T2DM patients than those in healthy control subjects (16, $19,22,26)$. In another paper, it is reported that in obese participants (15), FNDC5 gene expression in muscle was significantly decreased in type 2 diabetes. Regarding insulin resistance, some studies reported a negative correlation with HOMA-IR (27), others positive correlation (17, 28-30).

In this present study, we intended to evaluate irisin concentration in different stages of glucose tolerance, from normal to newly diagnosed type 2 diabetes. We aim to see its relation to the markers of glucose intolerance and we expect to contribute to the ongoing debate on its involvement in the glucose metabolism.

\section{Material and Methods}

The study has included 152 individuals (53 male and 99 female). Patients with any comorbidity such as hepatic, renal, cardiovascular, chronic inflammatory or infectious diseases, gestational diabetes or malignity were excluded. All participants were questioned about their daily activity and medication used. The study was approved by the ethics committee of our hospital.

A standard 75-gram oral glucose tolerance test (OGTT) was performed, and the total group was divided into five subgroups according to the results of the test. The subgroups were defined based on the American Diabetes Association (ADA) criteria for the diagnosis and classification of diabetes $2010(31,32)$; (1) normal glucose tolerance (NGT): fasting plasma glucose $(\mathrm{FPG})<100$ $\mathrm{mg} / \mathrm{dl}$ and 2 hour postprandial glucose $(2 \mathrm{hPG})<140 \mathrm{mg} / \mathrm{dl}$; (2) isolated impaired fasting glucose (IFG): FPG 100-126 $\mathrm{mg} / \mathrm{dl}$ and $2 \mathrm{hPG}<140 \mathrm{mg} / \mathrm{dl}$; (3) isolated impaired glucose tolerance (IGT): FPG $<100 \mathrm{mg} / \mathrm{dl}$ and $2 \mathrm{hPG} \mathrm{140-199}$ $\mathrm{mg} / \mathrm{dl}$; (4) combined IFG/IGT: FPG 100-125 and 2hPG 140-199 mg/dl; (5) type 2 diabetes mellitus (T2DM): FPG $\geq 126 \mathrm{mg} / \mathrm{dl}$ or $2 \mathrm{hPG} \geq 200 \mathrm{mg} / \mathrm{dl}$.

Blood samples obtained from the patients were centrifuged, and serum samples were stored at $-80{ }^{\circ} \mathrm{C}$ until analyzed. Serum glucose values were measured by enzymatic UV test of AU 5800 hexokinase method (Beckman Coulter, Germany), triglycerides, total cholesterol, and HDL cholesterol were measured by AU 5800 enzymatic colorimetric test (Beckman Coulter, Germany). Insulin levels were analyzed by electrochemiluminescence immunoassay method by the Modular E170 analyzer (Roche Diagnostics, Switzerland). HbAlc was analyzed by the cation exchange high-performance chromatography (Variant II Turbo, Biorad, USA). Body mass index (BMI) was calculated body mass divided by the square of the body height (weight $/$ hieght $^{2}$ ) and expressed in units of $\mathrm{kg} / \mathrm{m}^{2}$. Homeostatic model assessment (HOMA-IR) is used to quantify insulin resistance and calculated as fasting serum glucose $(\mathrm{mg} / \mathrm{dl}) \mathrm{x}$ fasting serum insulin $(\mu \mathrm{U} / \mathrm{ml}) / 405$ and expressed as \%. The Irisin assay was performed by Biovendor Elisa kit (Cat. No.: RAG018R, Czech Republic). Sensitivity (limit of detection) of the assay declared by the manufacturer is $1 \mathrm{ng} / \mathrm{ml}$. Assay range is $0.001 \mu \mathrm{g} / \mathrm{ml}-5$ $\mu \mathrm{g} / \mathrm{ml}$, intraassay and interassay CV's are 4.86-8.19 $\mu \mathrm{g} / \mathrm{ml}$ and $8.27-9.67 \mu \mathrm{g} / \mathrm{ml}$, respectively.

Descriptive statistics' were presented as the mean \pm standard deviation (SD) and median (2.5-97.5 \%) as required. The comparison of the groups was done with independent samples Student's t or Mann-Whitney U test. Correlations between irisin and the other variables were determined by Pearson's correlation analysis. The normality of the data distribution was evaluated by Kruskal-Wallis test. Multiple regression analysis was performed to determine the relation between irisin and age, gender, body mass index (BMI), fasting glucose, fasting insulin, total cholesterol, HDL cholesterol, triglycerides levels. Values of $p<0.05$ were considered significant. The statistical analyses were performed with Medcalc version 15.2.2 statistical software.

\section{Results}

The total study group included 32 NGT, 29 IFG, 31 IGT, $31 \mathrm{IFG} / \mathrm{IGT}$ and 29 T2DM patients (aged from 19 to 78 ). The demographic and anthropometric characteristics and the results of the biochemical markers are displayed in Table 1 . The $p$ values $<0.05$ displayed on the table meant that there was a significant difference between the means of at least two of the subgroups, according to one way analysis of variance. There was not a significant difference between groups with regard to age, HOMA-IR, total cholesterol, and HDL levels. 
Table 1. Demographic and anthropometric characteristics and biochemical values of the groups. Data are shown as Mean \pm SD or Median (95\% CI for the median) as required, and $\mathrm{p}$ values between groups.

\begin{tabular}{|c|c|c|c|c|c|c|c|}
\hline & Total Group & NGT & IFG & IGT & IFG/IGT & T2DM & $\mathrm{p}$ values \\
\hline Number & 152 & 32 & 29 & 31 & 31 & 29 & \\
\hline Age (year) & $46.93 \pm 11.56$ & $43.5 \pm 12.84$ & $47.86 \pm 9.77$ & $47.55 \pm 12.47$ & $48.42 \pm 10.94$ & $47.52 \pm 11.41$ & 0.455 \\
\hline BMI $\left(\mathrm{kg} / \mathrm{m}^{2}\right)$ & $31.78 \pm 5.58$ & $29.46 \pm 5.59$ & $31.42 \pm 4.67$ & $33.38 \pm 6.58$ & $33.82 \pm 4.83$ & $30.80 \pm 5.07$ & $0.009^{*}$ \\
\hline Glucose 0 (mg/dl) & $100.87 \pm 10.72$ & $90.65 \pm 5.98$ & $107.07 \pm 6.65$ & $92.13 \pm 4.81$ & $108.80 \pm 6.49$ & $106.79 \pm 10.79$ & $<0.001^{*}$ \\
\hline Glucose 120 (mg/dl) & $\begin{array}{c}147(65.30 \text { to } \\
244.70)\end{array}$ & $\begin{array}{c}94 \text { (54.90 to } \\
134.10)\end{array}$ & $\begin{array}{c}110(61.90 \text { to } \\
136.875)\end{array}$ & $\begin{array}{c}155(120.95 \text { to } \\
194.175)\end{array}$ & \begin{tabular}{|c|}
$162(143.55$ to \\
$195.725)$ \\
\end{tabular} & $\begin{array}{c}218(197.225 \\
\text { to } 279.375)\end{array}$ & $<0.001^{*}$ \\
\hline Insulin $0(\mu \mathrm{U} / \mathrm{mL})$ & $\begin{array}{c}14(5.00 \text { to } \\
36.20)\end{array}$ & $\begin{array}{c}10.5(5.30 \text { to } \\
40.70)\end{array}$ & $\begin{array}{c}16(6.225 \text { to } \\
26.325)\end{array}$ & $\begin{array}{c}13(5.00 \text { to } \\
48.15)\end{array}$ & \begin{tabular}{|c|}
$17(5.275$ to \\
$36.80)$ \\
\end{tabular} & $\begin{array}{c}14(4.125 \text { to } \\
30.425)\end{array}$ & 0.276 \\
\hline Insulin $120(\mu \mathrm{U} / \mathrm{mL})$ & $\begin{array}{c}84 \text { (22.00 to } \\
304.60)\end{array}$ & $\begin{array}{c}39 \text { (13.30 to } \\
181.40)\end{array}$ & $\begin{array}{c}69(22.90 \text { to } \\
155.00)\end{array}$ & $\begin{array}{c}118(42.475 \text { to } \\
351.225)\end{array}$ & \begin{tabular}{|c|}
$110(29.125$ to \\
$357.40)$ \\
\end{tabular} & \begin{tabular}{|c|}
$98(42.275$ to \\
$227.125)$ \\
\end{tabular} & $<0.001 *$ \\
\hline HOMA-IR (\%) & $\begin{array}{c}3.565(1.14 \text { to } \\
8.793)\end{array}$ & \begin{tabular}{|c|}
2.33 (1.140 to \\
$9.485)$ \\
\end{tabular} & $\begin{array}{c}4.15(1.629 \text { to } \\
7.511) \\
\end{array}$ & $\begin{array}{c}3.08(1.050 \text { to } \\
10.335)\end{array}$ & \begin{tabular}{|c|}
$4.52(1.408$ to \\
$9.593)$ \\
\end{tabular} & \begin{tabular}{|c|}
$3.66(0.920$ to \\
$8.437)$ \\
\end{tabular} & $0.012 *$ \\
\hline$(\%)$ & $5.77 \pm 0.46$ & $5.56 \pm 0.39$ & $5.79 \pm 0.42$ & $5.70 \pm 0.49$ & $5.83 \pm 0.39$ & $5.97 \pm 0.51$ & $0.007 *$ \\
\hline Irisin $(\mu \mathrm{g} / \mathrm{ml})$ & $\begin{array}{c}2.27(0.958 \text { to } \\
5.286)\end{array}$ & \begin{tabular}{|c|}
$2.237(1.173$ to \\
$5.331)$ \\
\end{tabular} & $\begin{array}{c}1.99(0.831 \text { to } \\
4.240)\end{array}$ & $\begin{array}{c}2.49(1.011 \text { to } \\
4.875)\end{array}$ & \begin{tabular}{|c|}
$2.23(0.738$ to \\
$5.669)$ \\
\end{tabular} & \begin{tabular}{|c|}
$2.575(1.140$ to \\
$5.104)$
\end{tabular} & 0.466 \\
\hline T. Cholesterol (mg/dl) & $212.07 \pm 42.46$ & $204.34 \pm 49.63$ & $212.24 \pm 42.66$ & $218.13 \pm 36.62$ & $212.03 \pm 43.11$ & $214 \pm 40.439$ & 0.786 \\
\hline Triglycerides (mg/dl) & $\begin{array}{c}120.5(46.60 \text { to } \\
390.00)\end{array}$ & $\begin{array}{c}101.5(50.80 \text { to } \\
375.20)\end{array}$ & $\begin{array}{c}131 \text { (38.700 to } \\
300.775)\end{array}$ & $\begin{array}{c}121(53.025 \text { to } \\
538.625)\end{array}$ & \begin{tabular}{|c|}
$119(45.225$ to \\
$307.275)$ \\
\end{tabular} & $\begin{array}{c}138(52.875 \text { to } \\
745.300)\end{array}$ & 0.297 \\
\hline HDL (mg/dl) & $\begin{array}{c}47 \text { (31.30 to } \\
75.10)\end{array}$ & $\begin{array}{c}46(33.00 \text { to } \\
108.10)\end{array}$ & $\begin{array}{c}47(34.225 \text { to } \\
63.775)\end{array}$ & $\begin{array}{c}48(31.275 \text { to } \\
78.000)\end{array}$ & $\begin{array}{c}48(35.00 \text { to } \\
69.15)\end{array}$ & $\begin{array}{c}45(26.225 \text { to } \\
61.775)\end{array}$ & 0.561 \\
\hline
\end{tabular}

$* \mathrm{p}<0.05$

Besides the obvious difference between glucose, insulin, and $\mathrm{HbA1c}$ values, the only significant difference was found between the BMI values of the IGT and IFG/IGT groups with the NGT patients $(p<0.05)$. Irisin levels of the five groups did not show any significant difference.

The correlation analysis revealed that irisin negatively correlated with age and positively correlated with HDL $(p=0.049$ and $p=0.021$ respectively). Partial correlation with adjustment for age showed a significant positive correlation between irisin and glucose- 120 , insulin- 120 and $\operatorname{HbAlc}(\mathrm{p}=0.026, \mathrm{p}=0.35$ and $\mathrm{p}=0.034$ respectively) in addition to the positive correlation with HDL which remained significant $(\mathrm{p}=0.011)$. The correlation analysis results are given in Table 2 . A further adjustment for age and BMI did not present any other differences.

Table 2. Correlation between serum irisin concentrations and the other markers levels, and partial correlation after adjustment for age.

\begin{tabular}{|c|c|c|c|c|}
\hline & \multicolumn{2}{|c|}{ Correlation } & \multicolumn{2}{|c|}{ Partial correlation } \\
\hline & Correlation coefficient & $\begin{array}{c}\text { Significance } \\
\text { Level P }\end{array}$ & Correlation coefficient & Significance Level $\mathbf{P}$ \\
\hline Age (year) & -0.160 & $0.0493 *$ & & \\
\hline BMI $\left(\mathbf{k g} / \mathbf{m}^{2}\right)$ & 0.011 & 0.892 & 0.021 & 0.802 \\
\hline Glucose 0 (mg/dl) & 0.010 & 0.900 & 0.020 & 0.811 \\
\hline Glucose 120 (mg/dl) & 0.151 & 0.064 & 0.181 & $0.0258^{*}$ \\
\hline Insulin $0(\mu \mathrm{U} / \mathrm{mL})$ & 0.027 & 0.739 & -0.009 & 0.908 \\
\hline Insulin $120(\mu \mathrm{U} / \mathrm{mL})$ & 0.098 & 0.232 & 0.171 & $0.0353 *$ \\
\hline HOMA-IR (\%) & 0.031 & 0.706 & -0.005 & 0.952 \\
\hline HbA1c (\%) & 0.110 & 0.176 & 0.173 & $0.0337^{*}$ \\
\hline T. Cholesterol (mg/dl) & -0.008 & 0.926 & 0.084 & 0.304 \\
\hline Triglycerides (mg/dl) & -0.097 & 0.234 & -0.069 & 0.403 \\
\hline HDL (mg/dl) & 0.187 & $0.0208 *$ & 0.207 & $0.0107 *$ \\
\hline
\end{tabular}

Regression analysis revealed that only age and triglycerides $(p=0.004$ and $p=0.015)$ contributed significantly to the prediction of irisin. The other variables were not found to be related to irisin according to multiple correlation coefficient or p-values (Table 3). 
Table 3. Multiple regression analysis of serum irisin concentrations with the other markers levels.

\begin{tabular}{|c|c|c|c|c|c|}
\hline Independent variables & Coefficient & Std. Error & $r_{\text {partial }}$ & $\mathrm{t}$ & $\mathrm{P}$ \\
\hline (Constant) & 1.139 & & & & \\
\hline Age (year) & -0.025 & 0.009 & -0.238 & -2.900 & $0.0043^{*}$ \\
\hline BMI $\left(\mathrm{kg} / \mathrm{m}^{2}\right)$ & -0.002 & 0.019 & -0.009 & -0.104 & 0.917 \\
\hline Glucose 0 (mg/dl) & -0.011 & 0.019 & -0.047 & -0.551 & 0.583 \\
\hline Glucose 120 (mg/dl) & 0.003 & 0.002 & 0.116 & 1.383 & 0.169 \\
\hline Insulin $0(\mu \mathrm{U} / \mathbf{m L})$ & -0.063 & 0.108 & -0.050 & -0.587 & 0.559 \\
\hline Insulin $120(\mu \mathrm{U} / \mathrm{mL})$ & 0.002 & 0.002 & 0.086 & 1.024 & 0.308 \\
\hline HOMA-IR (\%) & 0.258 & 0.443 & 0.049 & 0.582 & 0.562 \\
\hline HbA1c (\%) & 0.386 & 0.218 & 0.148 & 1.772 & 0.079 \\
\hline T. Cholesterol (mg/dl) & -0.002 & 0.003 & -0.058 & -0.691 & 0.491 \\
\hline Triglycerides (mg/dl) & 0.000 & 0.001 & -0.014 & -0.170 & 0.866 \\
\hline HDL (mg/dl) & 0.024 & 0.010 & 0.204 & 2.466 & $0.0149 *$ \\
\hline
\end{tabular}

\section{Discussion}

Even though it is a newly discovered biomarker, irisin has been extensively studied during the recent years. The evidence on the relation of the circulating irisin levels and exercise or BMI $(4,33)$ arose plenty of expectations. Irisin was thought to be a beneficial tool not only in diagnosis but also in the treatment of various metabolic problems. There are a quite large number of clinical researches performed on the concentration of irisin in several metabolic conditions, such as obesity, insulin resistance, or diabetes. Unfortunately, these studies did not end up in consistent results. They revealed negative, positive or null associations with age $(16,25,28,29)$, BMI $(16,17,27,30)$, HOMA $(17,29,30)$, or T2DM $(16,20,25,26,29)$.

The main output of this present study was that the irisin levels of individuals with different stages of glucose intolerance showed no significant difference. We did not find a difference between the irisin results of NGT and diabetes patients. There was not any correlation between irisin levels and insulin resistance index, namely HOMA-IR. On the other hand, we observed a positive and significant correlation between circulating irisin concentrations and the levels of 120-minute glucose, 120-minute insulin, and $\mathrm{HbA1}$.

Some studies performed on various populations in the recent few years claimed that irisin levels were lower in T2DM patients than normal subjects $(16,20,26)$. Furthermore, very recently, Duran et al., have observed lower circulating irisin levels in type 2 diabetes patients. They also have found that a significant decrease in irisin levels begins at the level of both IFG + IGT and gradually decrease with progression of glucose intolerance (19). Our data did not support these reports. However, the results of our study were consistent with other studies claiming that irisin levels were not associated with T2DM $(25,29,34)$. We suppose that these discrepancies were partly due to the diverse study populations concerning the age, gender, body composition, activity, etc. Additionally, the effect of methodological factors should be kept in mind, because of the not fully validated enzyme immunoassays kits, the poor standardization of the methods, varying target epitopes among manufacturers and, possible presence cross-reacting proteins. $(35,36)$ The method problems also could explain the marked difference between the circulating irisin levels in the mentioned reports, from $0.204 \mu \mathrm{g} / \mathrm{ml}$ to $2.83 \mu \mathrm{g} / \mathrm{mL}(16,19)$.

The association of irisin secretion with insulin resistance has also been examined by various researchers last years. Most of the studies reported a positive correlation between irisin and HOMA values as an insulin resistance marker $(17,29,30)$. In this present study, we could not find an association between irisin and HOMA-IR values. However, we observed a positive correlation between irisin concentrations and 120-minute insulin and 120-minute glucose. There are also controversial results on this subject. The data of Boström et al. on high-fat fed mice, illustrated that even moderately increased levels of circulating irisin potently increases energy expenditure, and improves diet-induced insulin resistance (4). On the other hand, in their study of single nucleotide polymorphisms (SNPs), Staiger et al. reported that a common genetic variation in the human FNDC5 locus, encoding the irisin precursor, determines insulin sensitivity and revealed a negative association between FNDC5 expression and in vivo measures of insulin sensitivity (24). They postulated that the biological functions of irisin might differ in mice and humans. Our data support the findings of the latter and some other clinical reports that claimed a positive correlation between irisin and insulin levels (17, 18, 28-30). Further studies are recommended to elucidate whether irisin is an adaptive response to counteract the disturbances in metabolic homeostasis or irisin levels represent a promotor of such disturbances (35). The potential 
resistance to irisin in obesity leading to hyperinsulinemia and insulin resistance should also be considered on this issue.

Our study did not reveal a significant correlation between irisin and BMI, in concordance with some authors $(25,29)$. However, there is a pretty much evidence of the positive correlation between irisin and $\operatorname{BMI}(16,17,27,30)$. Crujeiras et al. hypothesized that adipose tissue may play a role in determining circulating FNDC5/irisin levels in co-operation with muscle and that the muscle/adipose secretion ratio is affected by the pathophysiological situation (37). This could be an explanation for the inconsistent results. On this subject, Sanchis et al. suggested further studies to enlighten the issues such as; [1] irisin levels associated with BMI, reflecting primarily muscle or fat mass, [2] irisin levels are related to other biological variations, [3] the differences observed only reflect inter-population or methodological variations and/or assay discrepancies (38).

The irisin levels of our population showed a negative correlation with age. This result was consistent with Ebert's report (29) but not with some others which found no correlation $(25,28)$ or a positive correlation $(16)$. We speculate that this discrepancy could be attributed to population or method variations.

The association of irisin values with lipid profile was studied in different populations, but the results were controversial. Some studies found a positive association between irisin levels and beneficial lipid profile. As an exemple, in one of the largest studies, Oelmann et al. observed a significantly inverse association between irisin concentration and total and LDL cholesterol in male subjects (40). Also, most of the studies have found positive correlation between irisin and HDL (29, 41. 42. $43,44)$. However, not all studies gave consistent results with total and LDL cholesterol and triglycerides. The relation of irisin with total and LDL cholesterol was positive in some studies $(16,29,42)$ and negative in some others $(40,43,44)$. Similarly, some studies showed a positive correlation $(16,42)$ between irisin and triglycerides, some others showed a negative one $(29,43$, 44). We suggest that these discrepancies arise from the size and the type of the populations selected, the comorbidities which affects the resuls. As seen in Table 2, we found a week negative correlation between irisin and total cholesterol and triglycerides, and a week positive correlation between irisin and HDL, which supported positive association between irisin levels and beneficial lipid profile. However, the significance level was very low. As the study population was neither large enough, nor was selected for lipid profile evaluation, we presumed that our results could not be a contribution to the literature.

\section{Study Limitations}

The study had a couple limitations. First of all, it could be based on a larger population that would render the statistical analysis more reliable. Second, the activity status was based on the statements of the subjects instead of a measurable tool.

\section{Conclusions}

The conclusive results of this study are that irisin is somehow associated with insulin resistance but does not show a significant difference with diabetes or different stages of insulin resistance. We suppose that the association of irisin with the metabolic status, although considerably examined so far, needs further researches since the results are still conflicting. As proposed by Novelle et al., more studies are necessary on the precise roles of different forms of FNDC5/irisin and its receptor and the signaling pathway which will allow a better understanding of irisin function (39). We modestly propose that the elimination of the methodological problems, the definition of the biological variation affecting circulating irisin levels, large population-based molecular or clinical studies on irisin function and its association with metabolic processes could help to solve the issues. Irisin, which is a promising molecule, would then be a diagnostic or even therapeutic tool in metabolic disturbances.

\section{REFERENCES}

[1] Febbraio MA, Pedersen BK. Contraction-induced myokine production and release: is skeletal muscle an endocrine organ? Exerc Sport Sci Rev 2005; 33:114-119.

[2] Duzova H. Skeletal muscle, myokines and health. Med Sci 2012; 1:211-231.

[3] Pedersen BK, Febbraio MA. Muscles, exercise and obesity: skeletal muscle as a secretory organ. Nat Rev Endocrinol. 2012 Apr 3;8(8):457-65.

[4] Boström P, Wu J, Jedrychowski MP, et al. A PGC1- $\alpha$-dependent myokine that drives brown-fat-like development of white fat and thermogenesis. Nature. 2012; 11;481(7382):463-8.

[5] Wenz T, Rossi SG, Rotundo RL, et al. Increased muscle PGC-1alpha expression protects from sarcopenia and metabolic disease during aging. Proc Natl Acad Sci U S A. 2009; 106:20405-20410.

[6] Cannon, J. Nedergaard. Brown adipose tissue: function and physiological significance. Physiol. Rev. 2004; 84:2.

[7] Huh JY, Panagiotou G, Mougios V, et al. FNDC5 and irisin in humans: I. Predictors of circulating concentrations in serum and plasma and II. MRNA expression and circulating concentrations in response to weight loss and exercise. Metabolism. 2012; 61:1725-1738. 
[8] Kraemer RR, Shockett P, Webb ND, et al. A transient elevated irisin blood concentration in response to prolonged, moderate aerobic exercise in young men and women. Horm Metab Res 2014; 46(2):150-154.

[9] Norheim F, Langleite TM, Hjorth M, et al. The effects of acute and chronic exercise on PGC- $1 \alpha$, irisin and browning of subcutaneous adipose tissue in humans. FEBS J. 2014 Feb; 281(3):739-49.

[10] Pekkala S, Wiklund PK, Hulmi JJ, et al. Are skeletal muscle FNDC5 gene expression and irisin release regulated by exercise and related to health? J Physiol. 2013 Nov 1; 591(Pt 21):5393-400.

[11] Hecksteden A, Wegmann M, Steffen A, et al. Irisin and exercise training in humans - results from a randomized controlled training trial. BMC Med. 2013 Nov 5; 11:235.

[12] Kurdiova T, Balaz M, Vician M, et al. Effects of obesity, diabetes and exercise on Fndc5 gene expression and irisin release in human skeletal muscle and adipose tissue: in vivo and in vitro studies. J Physiol. 2014; 592(Pt 5):1091-107.

[13] Timmons JA, Baar K, Davidsen PK, et al. Is irisin a human exercise gene? Nature. 2012 Aug 30; 488(7413).

[14] Roca-Rivada A, Castelao C, Senin LL et al. FNDC5/Irisin Is Not Only a Myokine but Also an Adipokine. PLoS One. 2013;11; 8 e60563

[15] Moreno-Navarrete JM, Ortega F, Serrano M, et al. Irisin is expressed and produced by human muscle and adipose tissue in association with obesity and insulin resistance. J Clin Endocrinol metab. 2013 Apr;98(4):E769-78.

[16] Liu JJ, Wong MD, Toy WC, et al. Lower circulating irisin is associated with type 2 diabetes mellitus. J Diabetes Complications. 2013; 27:365-369.

[17] Park KH, Zaichenko L, Brinkoetter M, et al. Circulating irisin in relation to insulin resistance and the metabolic syndrome. J Clin Endocrinol Metab. 2013 Dec; 98(12):4899-907.

[18] Stengel A, Hofmann T, Goebel-Stengel M, et al. Circulating levels of irisin in patients with anorexia nervosa and different stages of obesity-correlation with body mass index. Peptides. 2013 Jan; 39:125-30.

[19] Duran ID, Gülçelik NE, Ünal M, et al. Irisin levels in the progression of diabetes in sedentary women. Clin Biochem. 2015 Dec; 48(18):1268-72.

[20] Choi YK, Kim MK, Bae KH, et al. Diabetes Res Clin Pract. Serum irisin levels in new-onset type 2 diabetes. 2013 Apr; 100(1):96-101.

[21] Hou N, Han F, Sun X. The relationship between circulating irisin levels and endothelial function in lean and obese subjects. Clin Endocrinol (Oxf). 2015 Sep; 83(3):339-43.

[22] Alis R, Sanchis-Gomar F, Pareja-Galeano H, et al. Association between irisin and homocysteine in euglycemic and diabetic subjects. Clin Biochem. 2014 Dec; 47(18):333-5.

[23] Crujeiras AB, Zulet MA, Lopez-Legarrea P, et al. Association between circulating irisin levels and the promotion of insulin resistance during the weight maintenance period after a dietary weight lowering program in obese patients. Metabolism. 2014 Apr; 63(4):520-31.

[24] Staiger H, Böhm A, Scheler M, et al. Common genetic variation in the human FNDC5 locus, encoding the novel muscle-derived 'browning' factor irisin, determines insulin sensitivity. PLoS One. 2013 Apr 25; 8(4):e61903.

[25] Sanchis-Gomar F, Alis R, Pareja-Galeano H, et al. Circulating irisin levels are not correlated with BMI, age, and other biological parameters in obese and diabetic patients. Endocrine. 2014 Aug; 46(3):674-7.

[26] Zhang HJ, Zhang XF, Ma ZM, et al. Irisin is inversely associated with intrahepatic triglycerides content in obese adults. J Hepatol. 2013 Sep; 59(3):557-62.

[27] Al-Daghri NM, Alkharfy KM, Rahman S, et al. Irisin as a predictor of glucose metabolism in children: sexually dimorphic effects. Eur J Clin Invest. 2014 Feb; 44(2):119-24.

[28] Reinehr T, Elfers C, Lass N, et al. Irisin and its relation to insulin resistance and puberty in obese children: a longitudinal analysis. J Clin Endocrinol Metab. 2015 May; 100(5):2123-30.

[29] Ebert T, Focke D, Petroff D, et al. Serum levels of the myokine irisin in relation to metabolic and renal function. Eur J Endocrinol. 2014 Mar 8; 170(4):501-6.

[30] Moreno M, Moreno-Navarrete JM, Serrano M, et al. Circulating irisin levels are positively associated with metabolic risk factors in sedentary subjects. PLoS One. 2015 Apr 21; 10(4):e0124100.

[31] Standards of Medical Care in Diabetes-2012. Diabetes Care 2012;35 (supplement 1):s64-s71.

[32] Nathan DM, Davidson MB, Defronzo RA, et al. Impaired Fasting Glucose and Impaired Glucose Tolerance. Implications for care. Diabetes Care 2007;30(3):753-759.

[33] Pardo M, Crujeiras A.B, Ami M. et al. Association of irisin with fat mass, resting energy expenditure, and daily activity in conditions of extreme body mass index. International Journal of Endocrinology, 2014; 2014:857270.

[34] Yang M, Chen $P$, Jin $H$, et al. circulating levels of irisin in middle-aged first-degree relatives of type 2 diabetes mellitus - correlation with pancreatic $\beta$-cell function. Diabetol Metab Syndr. 2014 Dec 5; 6(1):133

[35] Crujeiras AB, Pardo M, Casanueva FF. Irisin: 'fat' or artefact. Clinical Endocrinology 2015, 82:467-474.

[36] Eckardt K, Görgens SW, Raschke S, et al. Myokines in insulin resistance and type 2 diabetes. Diabetologia 2014; 57:1087-1099.

[37] Crujeiras AB, Pardo M, Arturo RR, et al. Longitudinal variation of circulating irisin after an enerjy restriction-induced weight loss and following weight regain in obese men and women. Am J Hum Biol. 2014 Mar-Apr; 26(2):198-207.

[38] Sanchis-Gomar F, Alis R, Pareja-Galeano H, et al. Inconsistency in circulating irisin levels: What is really happening? Horm Metab Res. 2014; 46:591-6.

[39] Novelle MG, Contreras C, Romero-Picó A, et al. Irisin, two years later. Int J Endocrinol. 2013; 2013:746281. 
[40] Oelman S, Nauck M, Völzke H, et al. Circulating Irisin Concentrations Are Associated with a Favourable Lipid Profile in the General Population. PLoS One. 2016 Apr 29; 11(4):e0154319.

[41] Buscemi S, Corleo D, Vasto $\mathrm{S}^{3}$, et al. Factors Associated with Circulating Concentrations of Irisin in the General Population Cohort of the ABCD Study. Int J Obes (Lond). 2018 Mar; 42(3):398-404.

[42] Jang HB, Kim HJ, Kang JH, et al. Association of circulating irisin levels with metabolic and metabolite profiles of Korean adolescents. Metabolism. 2017 Aug; 73:100-108.

[43] Liu BW, Yin FZ, Qi XM, Fan DM, Zhang Y. The Levels of Serum Irisin as a Predictor of Insulin Resistance in Han Chinese Adults with Metabolically Healthy Obesity. Clin Lab. 2017 May 1; 63(5):881-886.

[44] Wen MS, Wang CY, Lin SL, Hung KC. Decrease in irisin in patients with chronic kidney diesase. PLoS One 2013; 8(5): e64025.

i The authors have no conflict of interest to declare. 